\title{
The Effect of Hydroxychloroquine On Ventricular
}

\section{Repolarization Parameters Jtc And Jtpc Intervals In}

\section{Patients With Covid-19}

\author{
Önder Bilge ${ }^{1 *}$, Murat Çap ${ }^{1}$, Ferhat Işık ${ }^{1}$, Rojhat Altindag ${ }^{1}$, Abdurrahman Akyüz $^{1}$, Ümit \\ Inci $^{1}$, Burhan Aslan ${ }^{1}$, İlyas Kaya ${ }^{1}$, Mehmet Şahin Adıyaman ${ }^{1}$, Nesim Aladağ ${ }^{2}$, Halil Akın ${ }^{3}$, Bernas \\ Altıntaş ${ }^{1}$ \\ ${ }^{1}$ Department of Cardiology, University of Health Sciences Gaz̧i Yaşargil Education and Research Hospital, Diyarbakur, \\ Turkey \\ ${ }^{2}$ Department Of Cardiology,yüzüncü Yıl University Faculty Of Medicine, Van, Turkey \\ ${ }^{3}$ Department of Cardiology, Sinop State Hospital, Sinop, Turkey
}

\begin{abstract}
JT and JTp intervals are among the ventricular repolarization parameters and prolongation, just as the QT interval is related to ventricular arrhythmias. This study aims to examine the effect of hydroxychloroquine on JTc and JTpc intervals in individuals hospitalized with COVID-19.

We involved 130 COVID-19 patients divided into two groups as the hydroxychloroquine treatment group and the control group, in this study. ECGs of the patients were recorded at admission and after a median of 48 hours following the initiation of hydroxychloroquine treatment. Then, QTc, JTc, and JTpc intervals were measured.

Patients' average age was 48 (37-66 IQR), and 53\% of the individuals were female. The median basal JTpc interval was 215 ms(195-230 IQR), while JTpc-Day 3 was $220 \mathrm{~ms}(195-238 \mathrm{IQR})$. The median basal JTc interval was $320 \mathrm{~ms}(301-334 \mathrm{IQR}$ ), while JTc-Day 3 was $328 \mathrm{~ms}(306-343 \mathrm{IQR})$. JTpc and JTc interval in the hydroxychloroquine group have prolonged significantly $(\mathrm{p}<0.001)$, while there were no significant changes in the control group for both values $(\mathrm{p}>0.05)$.

There was a significant prolongation in the multivariable linear regression analysis in JTc-Day 3 and JTpc-Day 3 after hydroxychloroquine treatment. $(\beta=-2.589,95 \% \mathrm{CI}, 2.384-17.894, \mathrm{p}=0.011, \beta=2.195,95 \% \mathrm{CI}, 759-14.752, \mathrm{p}=0.030$, respectively).

In this study, we found significant prolongation in JTc and JTpc intervals in the patients who take hydroxychloroquine treatment.
\end{abstract}

Keywords: COVID-19, hydroxychloroquine, JTc interval, JTpc interval

\section{Introduction}

Coronavirus Disease-2019 (COVID-19), caused by Severe Acute Respiratory Syndrome-Coronavirus-2 (SARS-CoV-2), was first reported in China on December 31; 2019, and spread rapidly all over the world within a few months, leading to the declaration of a pandemic by the World Health Organization (WHO) (1-3). It leads to the involvement of many tissues and organs, especially the lungs. SARS-CoV-2 may cause myocardial damage and arrhythmias by prolonging the QT interval depending on the primary effect of the disease and the drugs used in treatment (4-6).
The QT interval reflects ventricular electrical activity called depolarization and repolarization. Previous studies have shown that hydroxychloroquine used in COVID-19 patients can cause prolongation in the QT interval and related fatal arrhythmias such as torsades de pointes (6-8). JT and JTp intervals are among the parameters of ventricular repolarization. Studies show that prolongation of the ventricular repolarization time causes an increase in ventricular fibrillation risk $(9,10)$. The JT interval can be used as an alternative to QT in patients with wide QRS. Some studies have shown that the JTp interval is a better parameter than the QT

*Corresponding Author: Önder Bilge, Department of Cardiology, SBU Diyarbakır Gazi Yaşargil Education and Research Hospital, Diyarbakir, Turkey

E-Mail: onder-bilge@hotmail.com, phone: +90 (412) 2580060

ORCID ID: Önder Bilge: 0000-0001-8214-972X, Murat Çap: 0000-0003-1164-2124, Ferhat Iş1k: 0000-0002-1438-3327, Rojhat Altindag: 0000-0002-2479-9318, Abdurrahman Akyüz: 0000-0003-2491-6593, Ümit Inci: 0000-0002-7412-1222, Burhan Aslan: 0000-0002-89947414, İlyas Kaya: 0000-0003-1998-6722, Mehmet Şahin Adıyaman: 0000-0003-2817-0629, Nesim Aladağ: 0000-0003-2346-1152, Halil Akın: 0000-0002-6651-7403, Bernas Altıntaş: 0000-0003-1662-3061

Received: 17.06.2021, Accepted: 07.12.2021 
interval in determining the torsadogenic effects of drugs (11-13). There is no study on the effect of hydroxychloroquine on JT and JTp intervals. We aimed to examine the effect of hydroxychloroquine on JTc and JTpc intervals in patients hospitalized with a COVID-19 diagnosis in this study.

\section{Materials and Methods}

This study was a single-centered retrospective observational study. COVID-19 patients hospitalized in our hospital from April 15 to May 30, 2020 were screened. After the exclusion criteria, the study included 130 patients with COVID-19. The diagnosis of the patients was confirmed with Real-time reverse transcriptasepolymerase chain reaction (RT-PCR) positivity. The patients with negative RT-PCR results, receiving azithromycin treatment, with atrial fibrillation and pacemaker rhythm, and without basal and day-3 ECG records were excluded from the study. The patients were divided into two groups as those who received hydroxychloroquine treatment $(n=74)$ and those who did not taking $(\mathrm{n}=56)$.

The sociodemographic and clinical features, and laboratory parameters of patients were collected from the electronic database of the hospital. ECGs of patients were recorded at admission and after a median of 48 hours following the initiation of hydroxychloroquine treatment. The ECGs recorded at admission were called Day-1, and the ECGs recorded after a median of 48 hours were named Day-3.

The loading dose for hydroxychloroquine was 400 mg twice a day on the first day and then as a maintenance dose of $200 \mathrm{mg}$ twice a day for 4 days.

Myocardial damage was defined as at least one cardiac troponin value above the 99th percentile upper reference limit. Existing at least 2 of the 4 systemic inflammatory response syndrome (SIRS) criteria (temperature $>38^{\circ} \mathrm{C}$ or $<36{ }^{\circ} \mathrm{C}$, heart rate $>90$, respiratory rate $>20$ or $\mathrm{PaCO}_{2}<32 \mathrm{~mm} \mathrm{Hg}$, WBC $>12000 / \mathrm{mm}^{3}$ or $<4000 / \mathrm{mm}^{3}$ or $>10 \%$ bands) was accepted as SIRS. $(6,14)$.

Electrocardiographic Evaluation: ECG (Schiller, Germany-Bavaria and Nihon Kohden, Japan-Tokyo) records were taken with 12 leads, at a speed of 25 millimeters/second and calibration of 10 millimeters/millivolt, and these records were transferred to the computer by means of a scanner. ECGs were examined by two independent cardiologists. In case of disagreement, they were reviewed by a third cardiologist. DII was used for analysis; in case of its unavailability, V5 was used. When both DII and V5 leads could not be evaluated, the remaining leads were examined

QT interval is the time from the beginning of the QRS complex to the end of the $\mathrm{T}$ wave. The time from the end of the QRS complex and the beginning of the J point to the end of the $\mathrm{T}$ wave is defined as the JT interval. The JTp interval is the time from the end of the QRS complex and the beginning of the $J$ point to the peak of the T wave. QT, JT, and JTp intervals were determined by using heart rate with the Bazett formula. $(\mathrm{QTc}=\mathrm{QT} / \sqrt{ } \mathrm{R}-\mathrm{R}, \mathrm{JTc}=\mathrm{JT} / \sqrt{-} \mathrm{R}-\mathrm{R}, \mathrm{JTpc}=$ $\mathrm{JTp} / \sqrt{-} \mathrm{R}-\mathrm{R})$

Permission was obtained from the Ministry of Health for this study, and the ethics committee approval was obtained from the local institutional ethics committee. Helsinki Declaration was complied within our study.

Statistical Analysis: SPSS 24 program was used for database and statistical analysis. The ShapiroWilks test were used to verify the normal distribution of datas. Since continuous variables did not show normal distribution, they were given as median and 25-75\% (IQR). Categorical variables were expressed as percentages. The Mann-Whitney U test was used for the continuous variables and the Chi-Square test or Fisher Exact Chi-Square for categorical variables for bivariate comparisons. The Wilcoxon rank test was used to compare paired repeated measures of independent groups. Multivariable linear regression analysis was used to assess the association of JTc-Day 3 and JTpc-Day 3 with clinical parameters (age, gender, CHF, hydroxychloroquine, favipiravir, potassium, calcium, SIRS, myocardial injury, loop diuretic b blocker). For statistical significance, $\mathrm{p}$ $<0.05$ was taken.

\section{Results}

The study included 130 individuals with COVID19 diagnosed by RT-PCR. The median age of the patients was 48 (37-66 IQR), and $53 \%$ of the individuals were female, while $47 \%$ were male. The demographic, clinical, and laboratory parameters of the individuals are shown in Table 1.

The median basal QTc interval was 431 ms (415-452 IQR), while QTc-Day 3 was 435 ms (419-456 IQR). In the hydroxychloroquine group, a median prolongation of $9 \mathrm{~ms}$ (-7-23 IQR) was observed in the QTc interval, and this prolongation was statistically significant $(p=0.004)$. In the control group, the median of the QTc interval was 
Table 1. Demographic Characteristics, Clinical and Laboratory Findings of The Patients

\begin{tabular}{|c|c|c|c|c|}
\hline & $\begin{array}{c}\text { Total } \\
(\mathrm{n}=130)\end{array}$ & $\begin{array}{l}\text { HCQ Group } \\
(\mathrm{n}=74)\end{array}$ & $\begin{array}{l}\text { Control Group } \\
(n=56)\end{array}$ & $\begin{array}{c}\mathrm{p} \\
\text { value }\end{array}$ \\
\hline Age, years & $48(37-66)$ & $46(31-66)$ & $50(39-66)$ & 0.156 \\
\hline Gender (female), n (\%) & $69(53 \%)$ & $36(49 \%)$ & $33(59 \%)$ & 0.245 \\
\hline Hypertension, n (\%) & $31(24 \%)$ & $15(20 \%)$ & $16(29 \%)$ & 0.372 \\
\hline Diabetes mellitus, n (\%) & $25(19 \%)$ & $12(16 \%)$ & $13(23 \%)$ & 0.437 \\
\hline Smoking, n (\%) & $38(29 \%)$ & $20(27 \%)$ & $18(32 \%)$ & 0.660 \\
\hline Congestive heart failure, $\mathrm{n}(\%)$ & $3(2 \%)$ & $1(1 \%)$ & $2(4 \%)$ & 0.577 \\
\hline Coronary artery disease, $\mathrm{n}(\%)$ & $14(11 \%)$ & $7(10 \%)$ & $7(13 \%)$ & 0.789 \\
\hline $\begin{array}{l}\text { Chronic respiratory disease, } \mathrm{n} \\
(\%)\end{array}$ & $8(6 \%)$ & $6(8 \%)$ & $2(4 \%)$ & 0.465 \\
\hline Myocardial injury, n (\%) & $5(4 \%)$ & $3(4 \%)$ & $2(4 \%)$ & 0.887 \\
\hline SIRS criteria $\geq 2, \mathrm{n}(\%)$ & $33(25 \%)$ & $18(24 \%)$ & $15(27 \%)$ & 0.908 \\
\hline Length of hospital stay, dats & $6(5-9)$ & $6(5-9)$ & $6(5-10)$ & 0.842 \\
\hline $\begin{array}{l}\text { Intensive care unit admission, } \\
\mathrm{n}(\%)\end{array}$ & $12(9 \%)$ & $7(10 \%)$ & $5(9 \%)$ & 0.943 \\
\hline Loop diuretic use, $\mathrm{n}(\%)$ & $3(2 \%)$ & $1(1 \%)$ & $2(4 \%)$ & 0.577 \\
\hline Temperature, ${ }^{\circ} \mathrm{C}$ & $37(36.7-37.5)$ & $37.1(36.6-37.5)$ & $37.1(36.7-37.6)$ & 0.633 \\
\hline Systolic blood pressure, $\mathrm{mmHg}$ & $110(105-120)$ & $110(100-120)$ & $110(106-120)$ & 0.442 \\
\hline Diastolic blood pressure, $\mathrm{mmHg}$ & $70(70-80)$ & $70(70-80)$ & $70(69-80)$ & 0.702 \\
\hline White blood cell, 103/uL & $6.06(4.57-7.49)$ & $6.31(4.79-8.46)$ & $5.62(4.52-7.02)$ & 0.151 \\
\hline Hemoglobin, $\mathrm{g} / \mathrm{dL}$ & $12.6(13.5-14.9)$ & $13.7(12.6-14.9)$ & $13.4(12.6-14.9)$ & 0.703 \\
\hline C-reactive protein, $\mathrm{mg} / \mathrm{L}$ & $11(2-47)$ & $6.1(2-47)$ & $27(4-49)$ & 0.015 \\
\hline Procalsitonin, $\mathrm{ng} / \mathrm{mL}$ & $0.06(0.04-0.1)$ & $0.05(0.04-0.1)$ & $0.08(0.05-0.11)$ & 0.98 \\
\hline $\mathrm{D}$-dimer, $\mathrm{ng} / \mathrm{mL}$ & $169(114-283)$ & $163(97-259)$ & $192(120-316)$ & 0.246 \\
\hline Creatinine, $\mathrm{mg} / \mathrm{dl}$ & $0.79(0.71-0.96)$ & $0.77(0.69-0.94)$ & $0.8(0.71-1.14)$ & 0.063 \\
\hline Albumin, $\mathrm{g} / \mathrm{L}$ & $39(36-43)$ & $42(38-45)$ & $37(34-40)$ & $<0.001$ \\
\hline Total calcium, mg/dL & $8.7(8.2-9.1)$ & $8.8(8.5-9.1)$ & $8.55(8-8.97)$ & 0.033 \\
\hline Corrected calcium, mg/dL & $8.67(8.42-8.96)$ & $8.61(8.35-8.87)$ & $8.72(8.48-9.18)$ & 0.076 \\
\hline Potassium, $\mathrm{mmol} / \mathrm{L}$ & $4.0(3.8-4.3)$ & $4.0(3.8-4.3)$ & $4.1(3.7-4.3)$ & 0.832 \\
\hline
\end{tabular}

Continuous variables are presented given as median (interquartile range) and categorical variables were expressed as number SIRS:Systemic inflammatory response syndrome

$-2 \mathrm{~ms}(-12-7 \mathrm{IQR})$, and this was not statistically significant $(\mathrm{p}=0.303)$.

The median basal JTpc interval was $215 \mathrm{~ms}(195-$ 230 IQR), while JTpc-Day 3 was 220 ms (195-238 IQR). In the hydroxychloroquine group, a median prolongation of $8 \mathrm{~ms}(0-23 \mathrm{IQR}, \mathrm{p}<0.001)$ was observed in the JTpc interval. In the control group, a change of $-3 \mathrm{~ms}(-15-13 \mathrm{IQR}, \mathrm{p}=0.940)$ was observed in the JTpc interval, which was not statistically significant. The median basal JTc interval was $320 \mathrm{~ms}$ (301-334 IQR), while JTc-Day 3 was $328 \mathrm{~ms}$ (306-343 IQR). In the hydroxychloroquine group, a median prolongation of 9 ms (-2-22 IQR) was observed in the JTc interval $(p$
$<0.001)$. In the control group, the median of the JTc interval was $-2 \mathrm{~ms}$ (-11-14 IQR), which was not statistically significant $(p=0.460)$. Electrocardiographic results of the patients are given in Table 2 .

In the multivariable linear regression analysis, the prolongation of the JTc was significantly correlated with the receiving hydroxychloroquine and the patients with SIRS. $(\beta=2.373,95 \%$ CI 1.316-14.589, $\mathrm{p}=$ $0.019, \beta=-2.589,95 \%$ CI 2.384-17.894, $\mathrm{p}=0.011, \beta=$ $10.423,95 \%$ CI $0.572-0.840, \mathrm{p}<0.001$, respectively). In addition, there was a significant relationship between JTpc-Day 3 and hydroxychloroquine, corrected calcium, and basal JTpc $(\beta=2.195$, 95\% CI 759$14.752, \mathrm{p}=0.030, \beta=-2.134,95 \%$ CI $-140.79-0.523$ 
Table 2. Electrocardiographic Findings of Patients

\begin{tabular}{lcccc}
\hline & Total $(\mathrm{n}=130)$ & HCQ Group $(\mathrm{n}=74)$ & Control $(\mathrm{n}=56)$ & p value \\
\hline Heart rate admission, beat/min & $90(78-99)$ & $90(76-99)$ & $90(79-98)$ & 0.539 \\
Heart rate at 3rd day, beat/min & $80(73-88)$ & $82(73-89)$ & $80(73-89)$ & 0.871 \\
QRS duration admission, ms & $90(83-100)$ & $94(84-104)$ & $88(83-96)$ & 0.020 \\
QRS duration 3rd-day, ms & $92(86-104)$ & $97(86-104)$ & $90(85-100)$ & 0.131 \\
QT interval admission, ms & $358(340-377)$ & $357(340-378)$ & $358(340-374)$ & 0.778 \\
QT interval 3rd-day, ms & $375(358-400)$ & $380(362-402)$ & $370(355-393)$ & 0.132 \\
QTc interval admission, ms & $431(415-452)$ & $427(414-452)$ & $433(42-451)$ & 0.321 \\
QTc interval 3rd-day, ms & $435(419-456)$ & $436(418-465)$ & $435(420-447)$ & 0.255 \\
QTc interval delta, ms & $2(-9-17)$ & $9(-7-23)$ & $-2(-12-7)$ & 0.006 \\
JT interval admission, ms & $262(240-283)$ & $260(237-283)$ & $267(254-283)$ & 0.402 \\
JTp interval admission, ms & $175(151-196)$ & $170(148-194)$ & $182(161-196)$ & 0.022 \\
JT interval 3rd-day, ms & $280(264-305)$ & $281(262-305)$ & $278(265-307)$ & 0.880 \\
JTp interval 3rd-day, ms & $186(168-210)$ & $185(163-210)$ & $189(175-210)$ & 0.331 \\
JTc interval admisson, ms & $320(301-334)$ & $317(293-331)$ & $322(314-338)$ & 0.025 \\
JTpc interval admission, ms & $215(191-230)$ & $203(183-228)$ & $223(209-236)$ & $<0.001$ \\
JTc interval 3rd-day, ms & $328(306-343)$ & $329(304-342)$ & $328(309-344)$ & 0.929 \\
JTpc interval 3rd-day, ms & $220(195-237)$ & $219(191-238)$ & $221(206-238)$ & 0.293 \\
JTc interval delta, ms & $6(-6.18)$ & $9(-2.23)$ & $-2(-11.14)$ & 0.006 \\
JTpc interval delta, ms & $6(-8.20)$ & $8(1.23)$ & $-4(-15.13)$ & $<0.001$ \\
\hline
\end{tabular}

Continuous variables are presented given as median (interquartile range) JTc:Corrected JT interval, JTp: peak JT interval, JTpc: Corrected JTp, QTc:Corrected QT

$18.068, \mathrm{p}=0.035, \beta=11.677,95 \%$ CI $0.676-0.952, \mathrm{p}$ $<0.001$, respectively) (Table 3).

\section{Discussion}

Our study determined a significant prolongation in JTc and JTpc intervals after hydroxychloroquine treatment. Although COVID19 mainly causes pneumonia, it can also lead to heart damage. Even though myocardial damage is directly related to viral infection, it may also occur due to hypoxia caused by acute severe respiratory infection (15). Previous studies have shown that hydroxychloroquine can cause prolongation in the QT interval and malignant ventricular arrhythmias such as torsades de pointes $(16,17)$. Hydroxychloroquine causes prolonged ventricular repolarization by blocking the hERG potassium channel in cardiac myositis, thus prolonging the QT interval (18). In our study, a significant prolongation in the QT interval was observed in the group receiving hydroxychloroquine.

Although it is known that hydroxychloroquine treatment given in COVID-19 patients prolongs the QT and QTc intervals, there are no data or studies investigating the effect on JTc and JTPc intervals. It corresponds to the early repolarization phase of the JTp action potential. In a study by Oikarinon et al., the JTp interval was longer in coronary artery patients with susceptibility to ventricular fibrillation (19). Not all drugs that prolong QT are proarrhythmic (20). It has been found that pure hERG channel blockers are more arrhythmogenic compared to calcium and late sodium channel blockers. Simultaneous inhibition of late sodium or calcium channels together with the hERG potassium channel reduces the risk of torsades de pointes by shortening JTPc. Prolongation of JTp has been associated with an increased risk of arrhythmia $(12,13)$. As a result of investigations by the FDA (U.S. Food and Drug Administration), it has been determined that the JTP interval can help distinguish pure hERG potassium channel blockers $(21,22)$. In our study, we found a significant association between JTPc-Day 3 and hydroxychloroquine treatment. Patients who take hydroxychloroquine treatment had statistically longer JTpc intervals than the control group.

The JT interval is stated to be a more specific marker of repolarization than the QT interval in patients with wide QRS. The American Heart Association and the American College of Cardiology recommend measurement of the JT interval in the presence of a wide QRS complex (11). The QT interval consists of depolarization and repolarization phases and is affected 
Table 3. Multivariable linear regression analysis between JTc (model 1), JTpc (model 2) interval and clinical variable

\begin{tabular}{|c|c|c|c|c|c|c|}
\hline & \multicolumn{3}{|c|}{ Model 1} & \multicolumn{3}{|c|}{ Model 2} \\
\hline & $\begin{array}{l}\beta- \\
\text { coefficient }\end{array}$ & CI 95\% & $\mathrm{P}$ value & $\begin{array}{l}\beta- \\
\text { coefficient }\end{array}$ & CI $95 \%$ & $\mathrm{P}$ value \\
\hline Age(year) & 1.331 & $-0.061-0.310$ & 0.186 & $-0,500$ & $-0.236-0.141$ & 0.618 \\
\hline Gender (female) & 1.725 & $-0.864-12.520$ & 0.87 & 1.713 & $-0.965-13.319$ & 0.089 \\
\hline $\mathrm{CHF}$ & -1923 & $-56.516-0.828$ & 0.057 & -0.947 & $\begin{array}{l}-42.886- \\
15.136\end{array}$ & 0.345 \\
\hline SIRS criteria $\geq 2$ & 2.589 & $2.384-17.894$ & 0.011 & 1.705 & $-14.079-0.523$ & 0.035 \\
\hline Hydroxycholoroquine & 2.373 & $1.316-14.589$ & 0.019 & 2.195 & $0.759-14.752$ & 0.030 \\
\hline Myocardial injury & $-1,654$ & $-32.320-2.900$ & 0.101 & -0.658 & $\begin{array}{l}-23.749- \\
11.910\end{array}$ & 0.512 \\
\hline Loop diuretic & 0.316 & $-25.238-34.824$ & 0.752 & 0.328 & $\begin{array}{l}-25.313- \\
35.360\end{array}$ & 0.744 \\
\hline Beta blocker & 0.565 & $-8.527-15.337$ & 0.573 & 0.858 & $-6.843-17.295$ & 0.393 \\
\hline $\begin{array}{l}\text { Corected } \\
\text { Calcium }(\mathrm{mg} / \mathrm{dL})\end{array}$ & -1288 & $-11.067-2343$ & 0.200 & -2.134 & $\begin{array}{l}-14,079-- \\
0.523\end{array}$ & 0.035 \\
\hline $\operatorname{Potassium}(\mathrm{mmol} / \mathrm{L})$ & -1.019 & $-11.109-3562$ & 0.310 & 0.159 & $-6.843-8.040$ & 0.874 \\
\hline JTc admission (ms) & 10.423 & $0.572-0.840$ & $<0.001$ & & & \\
\hline JTpc admmision (ms) & & & & 11.677 & $0.676-0.952$ & $<0.001$ \\
\hline
\end{tabular}

CHF: Congestive Heart Failure; JTc: Corrected JT interval, JTpc: Corrected JTp interval SIRS: Systemic Inflammatory Response Syndrome; CI:Confidence Interval

by the QRS duration (23). However, the JT interval reflects only ventricular repolarization (24). The studies with prolongation in both the QTc and JTc intervals found that the risk of developing ventricular arrhythmia increased and was associated with sudden cardiac death (9, 25-27). In a study evaluating Post MI patients with ventricular conduction delay and sensitivity to ventricular tachycardia, the JT interval was longer than the control group (28). In our study, we observed a significant association between JTPc-Day 3 and hydroxychloroquine, as prolonged JTPc-Day 3 after hydroxychloroquine treatment. A study with COVID19 patients found that the SIRS criterion of $\geq 2$ was a predictor of QTc prolongation after hydroxychloroquine and/or azithromycin treatment $(6,13)$. In our study, in the multivariable linear regression analysis, a significant association was found in between the SIRS criterion of $\geq 2$ and JTc-Day 3, JTp-Day 3.

In previous studies, it has been stated that the JT interval can be used as an alternative to the QT interval in patients with wide QRS. Considering the results of our study, the JT interval in patients receiving hydroxychloroquine treatment, regardless of the QRS duration, can provide more accurate information about the evaluation of the repolarization time. Again, it may be more appropriate to use the JTp interval, which has been stated to be more valuable than the QT interval in predicting arrhythmic events in patients taking hydroxychloroquine treatment with hERG potassium channel blockage and known torsadogenic effect.

A significant prolongation was observed in QTc, JTc, and JTpc intervals in patients received hydroxychloroquine treatment. In addition to the QT interval, monitoring of these parameters may be useful in predicting the development of arrhythmias in COVID-19 patients receiving hydroxychloroquine therapy.

\section{Conflict of interest: None}

Funding: No funding

\section{References}

1. Wu Z, McGoogan JM. Characteristics of and Important Lessons From the Coronavirus Disease 2019 (COVID-19) Outbreak in China: Summary of a Report of 72314 Cases From the Chinese Center for Disease Control and Prevention. JAMA 2020; 323: 1239-1242.

2. World Health Organization. WHO DirectorGeneral's opening remarks at the media briefing on COVID-19-11 March 2020. https://www.who.int/dg/speeches/detail/wh o-directorgeneral-s-opening-remarks-at-the- 
Bilge et al / Impact of hydroxychloroquine on JTpc and JTc

media-briefing-on-covid19-11-march-2020. Accessed March 24, 2020.

3. World Health Organization. Statement on the second meeting of the International Health Regulations (2005) Emergency Committee regarding the outbreak of novel coronavirus (2019-nCoV). https://www.who.int/newsroom/detail/30-01-2020-statement-on-thesecond-meeting-of-theinternational-healthregulations-(2005)-emergency-committeeregarding-the-outbreak-of-novel-coronavirus(2019-ncov). Accessed March 24, 2020.

4. Mitrani RD, Dabas N, Goldberger JJ. COVID-19 cardiac injury: Implications for long-term surveillance and outcomes in survivors. Heart Rhythm 2020; 17: 1984-1990.

5. Kang Y, Chen T, Mui D, at al. Cardiovascular manifestations and treatment considerations in COVID-19. Heart 2020; 106: 1132-1141.

6. Mercuro NJ, Yen CF, Shim DJ, at al. Risk of QT Interval Prolongation Associated With Use of Hydroxychloroquine With or Without Concomitant Azithromycin Among Hospitalized Patients Testing Positive for Coronavirus Disease 2019 (COVID-19). JAMA Cardiol 2020; 5: 1036-1041.

7. Cortegiani A, Ingoglia G, Ippolito M, Giarratano A, Einav S. A systematic review on the efficacy and safety of chloroquine for the treatment of COVID-19. J Crit Care 2020; 57: 279-283.

8. World Health Organization. The cardiotoxicity of antimalarials: Malaria Policy Advisory Committee Meeting. Published March 24 2017.https://www.who.int/malaria/mpac/mp ac-mar2017-erg-cardiotoxicity-report-session2;

9. Priori SG, Aliot E, Blømstrom-Lundqvist C, et al.. Task Force on Sudden Cardiac Death, European Society of Cardiology. Europace. 2002; 4: 3-18.

10. Murabayashi T, Fetics B, Kass D, Nevo E, Gramatikov B, Berger RD. Beat-to-beat QT interval variability associated with acute myocardial ischemia. J Electrocardiol 2002; 35: 19-25.

11. Rautaharju PM, Surawicz B, Gettes LS, at al; American Heart Association Electrocardiography and Arrhythmias Committee, Council on Clinical Cardiology; American College of Cardiology Foundation; Heart Rhythm Society. AHA/ACCF/HRS recommendations for the standardization and interpretation of the electrocardiogram: part IV: the ST segment, $\mathrm{T}$ and $\mathrm{U}$ waves, and the QT interval: a scientific statement from the American Heart Association Electrocardiography and Arrhythmias Committee, Council on Clinical Cardiology; the American College of Cardiology Foundation; and the Heart Rhythm Society.
Endorsed by the International Society for Computerized Electrocardiology. J Am Coll Cardiol 2009; 53: 982-991.

12. Johannesen L, Vicente J, Mason JW, Sanabria C, Waite-Labott K, Hong M, Guo P, Lin J, Sørensen JS, Galeotti L, Florian J, Ugander M, Stockbridge N, Strauss DG. Differentiating drug-induced multichannel block on the electrocardiogram: randomized study of dofetilide, quinidine, ranolazine, and verapamil. Clin Pharmacol Ther 2014; 96: 549558.

13. Johannesen L, Vicente J, Gray RA, at al. Improving the assessment of heart toxicity for all new drugs through translational regulatory science. Clin Pharmacol Ther 2014; 95: 501508.

14. Bone RC, Balk RA, Cerra FB, at al. Definitions for sepsis and organ failure and guidelines for the use of innovative therapies in sepsis. The ACCP/SCCM Consensus Conference Committee. American College of Chest Physicians/Society of Critical Care Medicine. Chest 1992; 101: 1644-1655.

15. Fan Y, Guo T, Lu Z. Myocardial Injury in COVID-19-Can We Successfully Target Inflammation?-Reply. JAMA Cardiol 2020; 5: 1070-1071.

16. Tönnesmann E, Kandolf R, Lewalter T. Chloroquine cardiomyopathy - a review of the literature. Immunopharmacol Immunotoxicol 2013; 35: 434-442.

17. Naqvi TZ, Luthringer D, Marchevsky A, Saouf R, Gul K, Buchbinder NA. Chloroquine-induced cardiomyopathyechocardiographic features. J Am Soc Echocardiogr 2005; 18: 383-387.

18. Naksuk N, Lazar S, Peeraphatdit TB. Cardiac safety of off-label COVID-19 drug therapy: a review and proposed monitoring protocol. Eur Heart J Acute Cardiovasc Care 2020; 9: 215-221.

19. Oikarinen L, Toivonen L, Viitasalo M. Electrocardiographic measures of ventricular repolarisation dispersion in patients with coronary artery disease susceptible to ventricular fibrillation. Heart 1998; 79: 554559.

20. Fenichel RR, Malik M, Antzelevitch C, at al; Independent Academic Task Force. Druginduced torsades de pointes and implications for drug development. J Cardiovasc Electrophysiol 2004; 15: 475-495.

21. Johannesen L, Vicente J, Mason JW, at al. Late sodium current block for drug-induced long QT syndrome: Results from a prospective clinical trial. Clin Pharmacol Ther 2016; 99: 214-223.

East J Med Volume:27, Number:1, January-March/2022 
22. Vicente J, Johannesen L, Mason JW, Crumb WJ, Pueyo E, Stockbridge N, Strauss DG. Comprehensive $\mathrm{T}$ wave morphology assessment in a randomized clinical study of dofetilide, quinidine, ranolazine, and verapamil. J Am Heart Assoc 2015; 4: e001615.

23. Crow RS, Hannan PJ, Folsom AR. Prognostic significance of corrected QT and corrected JT interval for incident coronary heart disease in a general population sample stratified by presence or absence of wide QRS complex: the ARIC Study with 13 years of follow-up. Circulation 2003; 108: 1985-1989.

24. Bihlmeyer NA, Brody JA, Smith AV, at al. ExomeChip-Wide Analysis of 95626 Individuals Identifies 10 Novel Loci Associated With QT and JT Intervals. Circ Genom Precis Med 2018; 11: e001758.
25. Bonnemeier H, Hartmann F, Wiegand UK, Bode F, Katus HA, Richardt G. Course and prognostic implications of QT interval and QT interval variability after primary coronary angioplasty in acute myocardial infarction. J Am Coll Cardiol 2001; 37: 44-50.

26. Vrtovec B, Starc V, Starc R. Beat-to-beat QT interval variability in coronary patients. J Electrocardiol 2000; 33: 119-125.

27. Vrtovec B, Starc V, Meden-Vrtovec H. The effect of estrogen replacement therapy on ventricular repolarization dynamics in healthy postmenopausal women. J Electrocardiol 2001; 34: 277-283.

28. Oikarinen L, Viitasalo M, Toivonen L. Dispersions of the QT interval in postmyocardial infarction patients presenting with ventricular tachycardia or with ventricular fibrillation. Am J Cardiol 1998; 81: 694-697. 